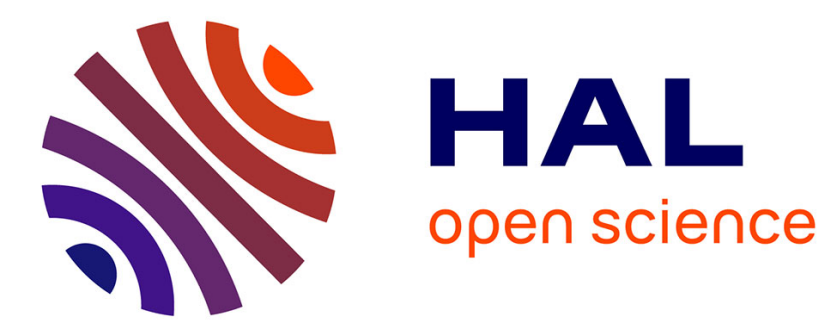

\title{
Screening of optimal structure among large-scale multi-state weighted k-out-of-n systems considering reliability evaluation
}

Yishuang Hu, Yu Lin, Yi Ding, Xingying Chen, Zhiguo Zeng

\section{To cite this version:}

Yishuang Hu, Yu Lin, Yi Ding, Xingying Chen, Zhiguo Zeng. Screening of optimal structure among large-scale multi-state weighted k-out-of-n systems considering reliability evaluation. Reliability Engineering and System Safety, 2021, 206, pp.107268. 10.1016/j.ress.2020.107268 . hal-03464080

\author{
HAL Id: hal-03464080 \\ https://hal.science/hal-03464080
}

Submitted on 11 Jan 2022

HAL is a multi-disciplinary open access archive for the deposit and dissemination of scientific research documents, whether they are published or not. The documents may come from teaching and research institutions in France or abroad, or from public or private research centers.
L'archive ouverte pluridisciplinaire HAL, est destinée au dépôt et à la diffusion de documents scientifiques de niveau recherche, publiés ou non, émanant des établissements d'enseignement et de recherche français ou étrangers, des laboratoires publics ou privés. 


\title{
Screening of Optimal Structure among Large-scale Multi- State Weighted k-out-of-n Systems Considering Reliability Evaluation
}

\author{
Yishuang $\mathrm{Hu}^{a}$, Yu Lin ${ }^{a}$, Yi Ding*a ${ }^{*}$, Xingying $\mathrm{Chen}^{b}$, Zhiguo Zeng ${ }^{c}$ \\ ${ }^{a}$ College of Electrical Engineering, Zhejiang University, Hangzhou, China \\ ${ }^{b}$ College of Energy and Electrical Engineering, Hohai University, Nanjing, China \\ 'EDF Foundation Chair on Systems Science and Energetic Challenge, CentraleSupelec, Universite Paris- \\ Saclay, Chatenay-Malabry, France
}

\begin{abstract}
Keen interest in the development and utilization of multi-state weighted $k$-out-of- $n$ systems (MSWKNS) has been currently observed. In real-life cases, considering that multiple different MSWKNS structures are provided beforehand, system designers need to select an optimal one among numbers of different structure designs. Thus, screening of the optimal MSWKNS structure is meaningful and critical. Moreover, the system reliability becomes an increasingly important factor that should be assessed in structure screening. However, when facing a large-scale MSWKNS whose number of states is enormous, exact reliability evaluation can be rather complicated. Meanwhile, due to the large number of system structure designs, the computational burden of screening can be very huge. To effectively select the optimal structure among enormous MSWKNSs under reliability constraints, an optimal structure screening method named as ordinal structure screening (OSS) is proposed. Ordinal optimization (OO) algorithm is adopted with a novel utilization of continuization discretization approximation (CDA) method. To shorten the reliability evaluation time, CDA is employed to approximate reliabilities. Based on the approximate reliabilities, OO is applied to reduce the number of system structures to $b$ evaluated, which accelerates the screening process. Illustrative results show that the proposed method appeals in improving computational efficiency with satisfactory accuracy performance.
\end{abstract}




\section{Keywords}

Multi-state weighted $k$-out-of- $n$ system; structure screening; ordinal optimization; continuization discretization approximation

\section{Acronyms}

MSWKNS multi-state weighted $k$-out-of- $n$ system

UGF universal generating function

OPC ordered performance curve

OSS ordinal structure screening

OO ordinal optimization

CDA continuization discretization approximate

\section{Notations}

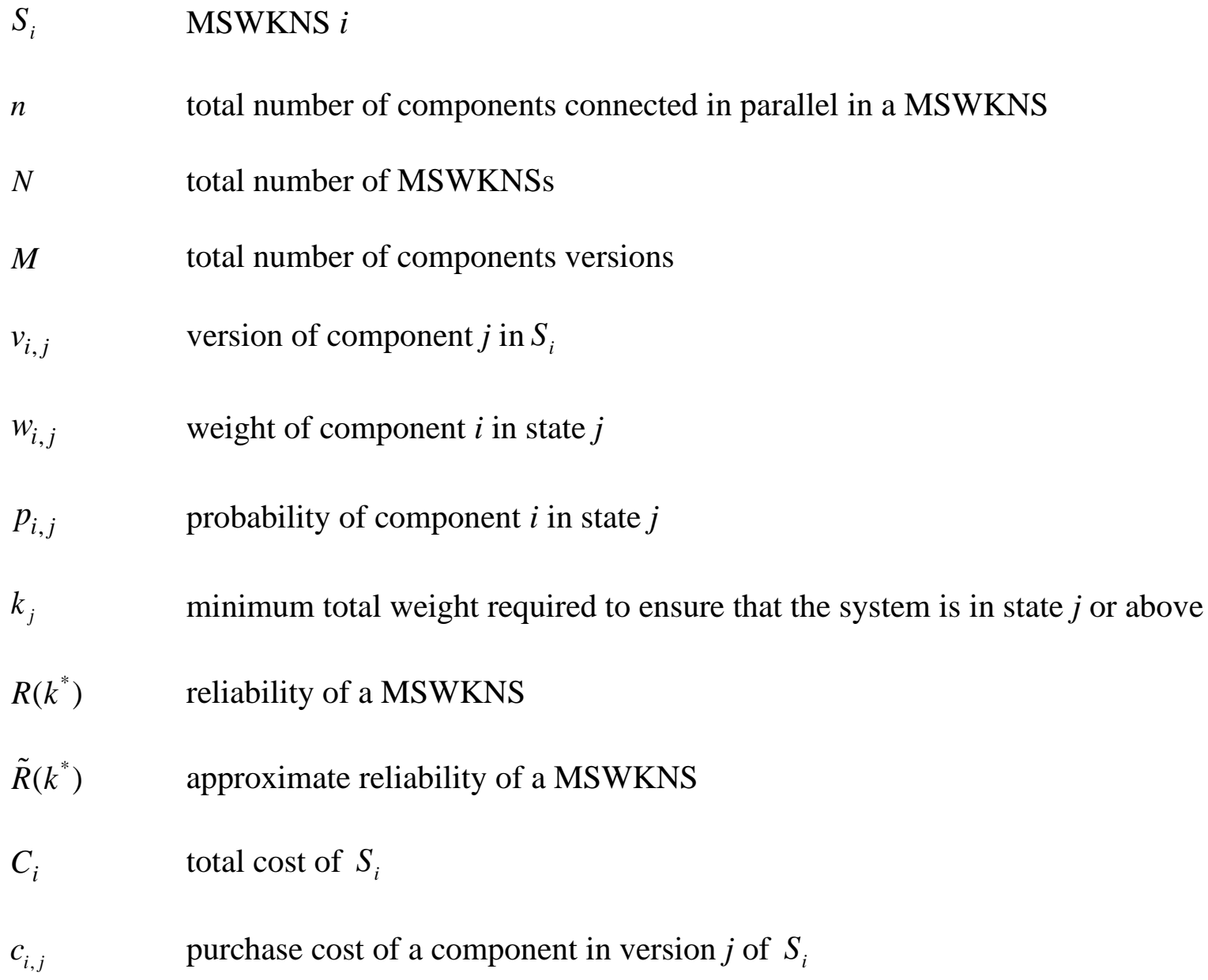




\section{Introduction}

Wide application of multi-state systems (MSSs) in reliability evaluation of real-life engineering systems has been observed [1]. In the MSS, the components and the systems have several performance levels. The system total performance can be evaluated by the contributions of each component at different states [2]. As one of the most typical MSS topologies, multi-state weighted $k$-out-of- $n$ system (MSWKNS) is widely accepted by every aspect of industry [2]. In a MSWKNS, component $i$ in state $j$ carries a performance weight of $w_{i, j}$. One of the basic conditions for the system to be working is that the total weight of all components is at least a predefined threshold value $k$. The structure of MSWKNS has been intensively investigated in recent researches [3].

Structure screening is one of the most important challenges that system designers encounter. In some real-life cases, there are multiple possible MSWKNS structure designs to satisfy different system needs. Each MSWKNS structure design has its unique characteristics, such as system cost. It is inevitable for system designers to efficiently select the optimal one among those structure designs. For example, there are 50 different MSWKNS structure designs in a planning of power systems. System designers need to select the optimal one which has minimal system cost [1]. Hence, screening of an optimal MSWKNS structure in terms of system cost has practical significance.

The structure screening of MSWKNSs should take different factors into account. Fpr example, the optimal system structure should be both economical and reliable [4], [5]. In addition to the system cost, maintaining a high reliability level of engineering systems is extremely important. For example, if the reliability of a generator does not meet the requirement, adequate electricity cannot be provided and the stable operation of power system will be affected. To ensure the reliability of a structure is no less than the required value, reliability evaluation of MSWKNSs is inevitable. In this paper, the structure screening can be 
regarded as a problem that needs to find the optimal option with the smallest system cost under the reliability constraints.

However, as the industry develops, the scale of engineering systems increases. In a largescale system, both the number of components and the system possible states are enormous [6], [7]. The great number of components and possible states makes reliability evaluation for large-scale systems extremely complex. Moreover, not only the scale of each system structure can be large, but also the number of possible structure designs can be numerous. It is inefficient to detailly and exactly calculate the system cost and reliability for each structure, especially for a structure screening containing plenty of large-scale structure designs. Considering the challenge of large-scale MSWKNS structures mentioned above, efficient structure screening among a wide variety of large-scale structures is urgent to be investigated.

Universal generating function (UGF) technique and recursive method are two primary algorithms for exact reliabilities evaluation of MSWKNSs. Reference [8] provided a recursive algorithm for reliability evaluation of MSWKNS. The UGF method was firstly introduced in [9] to reduce computational complexity for the MSS. By defining different operators, it can be applied to many different system structures [10], including $k$-out-of- $n$ structure. The Monte Carlo simulation (MCS) method [11], [12], and Markov process method [13] are also applicable in reliability evaluation. However, to evaluate the exact reliabilities of multiple large-scale MSWKNSs in structure screening, huge computational resource is required. In some real-life cases, the exact reliability is not necessary. The accuracy of reliability evaluation can be sacrificed to some extent for reducing the computational time. Therefore, considering the challenge of the large-scale MSWKNSs, an approximate reliability evaluation approach is needed.

Facing the difficulties of enormous searching space and exact reliability evaluation in structure screening, ordinal optimization $(\mathrm{OO})$ algorithm is adopted in this paper. This novel 
method is capable of effectively reducing the large searching space into a relatively small selected subset [14], [15], which can improve the computational efficiency. For example, reference [16] incorporated the OO into automation learning algorithm to improve the learning efficiency. The most important characteristics of OO is the consideration of "order" rather than the "value" during optimization [17], [18]. Therefore, an efficient performance approximation method to achieve a performance order of structures is inevitable. In this paper, continuization discretization approximation (CDA) method is utilized to evaluate the approximate reliabilities of MSWKNS structures. CDA can approximate the discrete probability distribution by a continuous Gaussian distribution and reduce the computational complexity [19] [20]. With the approximate reliabilities evaluated by CDA, OO can quickly narrow the searching space by comparing the reliability order first [15].

In this paper, an optimal structure screening method, referred as ordinal structure screening (OSS), is proposed, which consists of CDA, OO and UGF methods. OSS can effectively select the optimal one among multiple large-scale MSWKNS structure designs under reliability constraints. To reduce the reliability evaluation time, the approximate reliabilities of MSWKNSs is evaluated by CDA. Based on the pre-determined approximate reliabilities, OO can eliminate those large-scale MSWKNS structure designs with low reliabilities and largely minimize the number of designs employed in the final step. Thus, it can statistically improve the calculation efficiency. In structure screening, approximate reliability is unable to ensure that the reliability of a structure is no less than the required reliability. Thus, the exact reliability evaluation is indispensable. In the final step, UGF technique is used to evaluate the exact reliabilities for those pick-out MSWKNSs.

The major contributions of this paper are list as follows:

1) A novel analytical method in structure screening is proposed, which can efficiently and accurately select the optimal structure among multiple MSWKNSs. 
2) A new ordinal optimization method in structure screening with multiple MSWKNS designs is presented, which can restrict the large searching space into a small selected space.

3) An approximate reliability evaluation method in structure screening is developed with the combination of CDA and $\mathrm{OO}$.

The paper is structured as follows. Section 2 introduces the MSWKNS model and structure screening. Section 3 proposes the OSS method and its detailed explanation. Section 4 analyzes the performances of the proposed method by comparing with the existing techniques. Finally, the conclusion is presented in Section 5.

\section{Descriptions of MSWKNS and structure screening}

Descriptions of MSWKNS and structure screening are provided in this section. We assume that a MSWKNS consists of $n$ components connected in parallel. Different versions and numbers can be chosen for any given components. Different versions of components have different performance characteristics. Each component $i(i=1,2, \ldots, n)$ has $s_{i}$ different states, from totally failure state to perfectly functioning state. Let $w_{i, j}$ represent the performance weight of component $i$ in state $j\left(j=1,2, \ldots, s_{i}\right)$. Thus, the set of all performance weights of component $i$ is represented by $\bar{w}_{i}=\left\{w_{i, 1}, w_{i, 2}, \cdots, w_{i . s_{i}}\right\}$. Let the random variable $W_{i}$ represents the performance weight of component $i$, which takes value in the set $\bar{w}_{i}: W_{i} \in \bar{w}_{i}$. $p_{i, j}=\operatorname{Pr}\left(W_{i}=w_{i, j}\right)$ is the probability that $W_{i}$ is equal to $w_{i, j}$ (in state $j$ of component $i$ ) [3].

The system weight $W_{s}$ of the entire MSWKNS depends on the parallel interaction between components and on the distribution of the components' performances. The system performance level $W_{s}=f\left(W_{1}, W_{2}, \ldots, W_{n}\right)=\sum_{i=1}^{n} W_{i}$ is determined by the system structure function. The system is in state $j$ or above if $W_{s}$ is greater than or equal to a predefined threshold weight $k_{j}$. Let $\Phi$ represent the state of the system. We then have 
$\operatorname{Pr}(\Phi \geq j)=\operatorname{Pr}\left(W_{s} \geq k_{j}\right)$. Since state 0 is the worst state of the system, we have $\operatorname{Pr}(\Phi \geq 0)=1$.

The reliability of a MSWKNS $R\left(k^{*}\right)$ is the probability that the system weight is greater than or equal to $k^{*}$, which is expressed by $R\left(k^{*}\right)=\operatorname{Pr}\left(W_{s} \geq k^{*}\right)$ [3].

The goal of this work is to comprehensively select an optimal system structure which can satisfy different system requirements. Particularly, in real-life cases, to design an economical and reliable MSWKNS structure, enormous system structure designs are provided by system designers. Moreover, the structure characteristics of each MSWKNS, including the number and version of system components, are usually pre-determined. Based on that, system designers need to select the optimal system structure among multiple MSWKNS designs. An illustration of structure screening is given in Fig. 1.

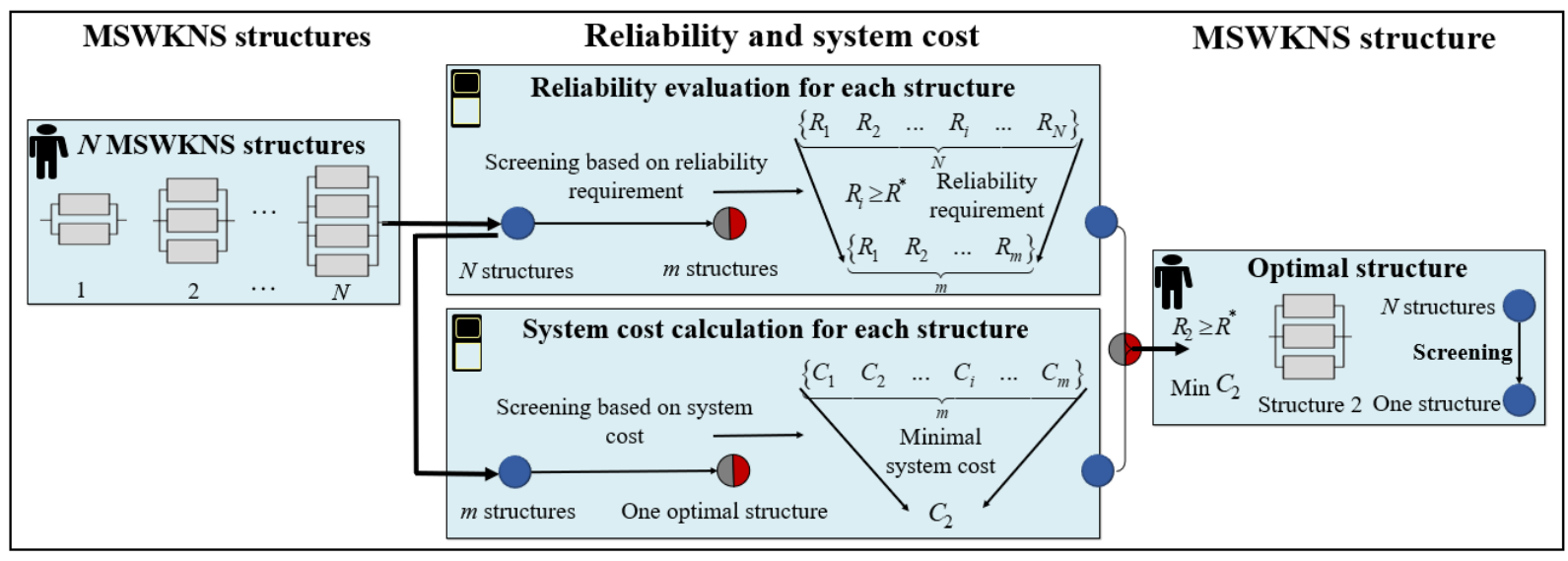

Fig. 1 Structure screening

Suppose there are $N$ MSWKNS structure designs. MSWKNS $S_{i}(i=1,2, \ldots, N)$ contains $n_{i}$ components. The components in $S_{i}$ can be divided into $M_{i}$ versions. Components from the same version have the same performance characteristics, such as component cost, performance weights and the corresponding probabilities. Assume that the $M_{i}$ versions of components in $S_{i}$ are denoted as $V_{i, j}\left(i=1,2, \ldots, N, j=1,2, \ldots, M_{i}\right)$. The total number of components in version $j V_{i, j}$ is $n_{i . j}$ and the cost for a component in $V_{i, j}$ is $c_{i, j}$. In this paper, 
the system cost is considered as the sum of total purchase costs for all components, which is the function of the number of components purchased simultaneously. Consequently, the system cost can be characterized as $C_{i}=\sum_{j=1}^{M_{i}}\left(c_{i, j} \cdot n_{i, j}\right)$. The reliability $R_{i}$ of structure $S_{i}$ can be assessed by reliability evaluation methods.

In structure screening, the reliability is served as the screening constraints. Among all the designs, the optimal structure is the most economical and reliable system whose system cost is minimal and its reliability is no less than the required value. As can be seen in Fig. 1, the framework of structure screening can be divided into 3 steps. The first step of structure screening is to evaluate the reliability $R_{i}$ for every MSWKNS $S_{i}(i=1,2, \ldots, N)$, then select the $m,(m \leq N)$ feasible MSWKNS structures into next step. The $m$ feasible structures are the MSWKNSs whose reliabilities are no less than the required reliability $R^{*}$. Finally, estimate the system cost $C^{\prime}{ }_{i}$ for every feasible MSWKNS $S_{i}^{\prime}(i=1,2, \ldots, m)$ and select the optimal structure which has minimal system cost. Through these three steps, the $N$ MSWKNS structure designs are screened into one optimal structure.

However, the computational burden for structure screening may be huge. For example, the number of possible MSWKNS structures can be large in structure screening. Suppose that there are $g$ different MSWKNS structure designs. Then, the system reliability has to be evaluated $g$ times, which requires huge computational resources to evaluate. Moreover, the exact reliability evaluation of a large-scale MSWKNS can be complicated. Taking reliability evaluation method UGF as an example, if a MSWKNS has $n$ components and each component has $k$ states, the computational complexity for this MSWKNS is $O\left(k^{n}\right)$. With the increasing number of components and states, the computational complexity can grow exponentially [21]. Therefore, the structure screening with exact reliability evaluation for multiple large-scale MSWKNS structure designs requires tremendous computational 
resources. To efficiently and accurately cope with the computational burden mentioned above, a novel structure screening framework named as OSS is presented in section 3.

\section{Formulation of OSS method}

In this section, OSS method is proposed to efficiently determine the optimal MSWKNS structure under reliability constraints. Suppose there are $N$ MSWKNS structure designs. Each system consists of $n$ components and $m$ states for each component. The computational complexity for structure screening together with exact reliability evaluation by UGF is $O\left(N \cdot m^{n}\right)$. Aiming at reducing the complexity for screening with numerous large-scale structure designs, OSS method is proposed. Firstly, reliability evaluation method utilized in OSS approximates discrete probability distribution by a continuous Gaussian distribution when the exact reliability is not available [19], whose computational complexity is much smaller than UGF. Secondly, the structure screening rule adopted in OSS efficiently cuts down the number of structure designs that need exact reliabilities evaluation. The procedures of OSS method are illustrated in Fig. 2.

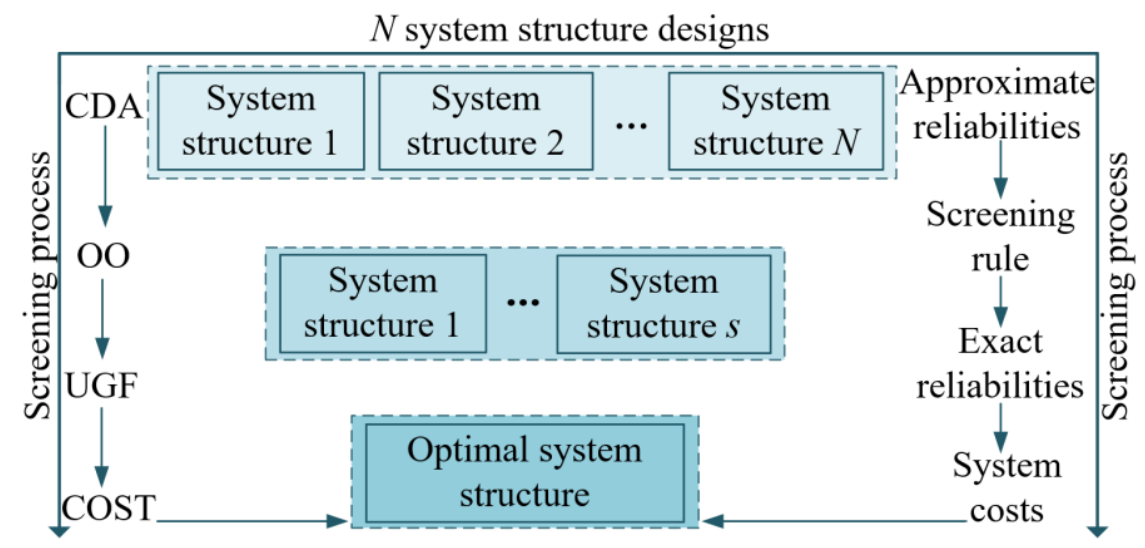

Fig. 2 OSS method

The OSS method consists of three algorithms, OO, CDA and UGF. CDA can evaluate the approximate reliabilities of MSWKNSs. Additionally, OO can eliminate the MSWKNS structure designs based on the approximate reliabilities. UGF is employed to assess the exact reliabilities of the remaining MSWKNS structures. The procedure of OSS in structure 
screening is presented as follows. Firstly, CDA is applied for evaluating the approximate reliabilities of MSWKNSs. Secondly, utilizing screening rule in OO and the approximate reliabilities evaluated by CDA, the top-s MSWKNS structures with highest approximate reliabilities are selected. The theory of OO ensures that the selected top- $s$ MSWKNS structures contain at least $k$ truly good structure designs with high probability [16]. The screening rule in $\mathrm{OO}$ can reduce the screening time by decreasing the number of structure designs. Thirdly, considering the reliability constraints, the exact reliabilities of selected top- $s$ MSWKNSs are evaluated by UGF technique. By comparing the exact reliabilities with required reliability, the feasible structures whose exact reliabilities meet the reliability constraints can be selected. Finally, comparing the system cost of each feasible structure, the optimal structure with minimum system cost can be screened. Particularly, the computational burden for the structure screening of MSWKNSs is dramatically reduced by the CDA and OO methods in the former steps.

\subsection{Approximate reliabilities of $N$ MSWKNSs}

This part introduces the technique to approximate MSWKNS reliability by applying the CDA in structure screening. With the increasing number of components and states, the number of terms considered in the reliability evaluation can grow exponentially [19]. The reliability evaluation complexity for a MSWKNS with $n$ components by UGF is $O\left(K^{n}\right)$, where $K$ is the number of states a component may have, while the computational complexity of CDA is $O(K n)$. Compared with UGF, the CDA is much more efficient [19]. Therefore, we apply CDA to evaluate the approximate reliability of a MSWKNS.

As mentioned in [19], it is a widely-employed assumption that the system components are independent. Thus, the random performance of these components $W_{i}(i=1,2, \ldots, n)$ are also assumed to be independent. Then, the simplified Lindeberg's central limit theory [20] can be applied in reliability evaluation of a MSWKNS. 
The random performance of system components $W_{i}(i=1,2, \ldots, n)$ are regarded as a sequence of independent discrete random variables. Their sum is the system performance weight $W_{s}=\sum_{i=1}^{n} W_{i}$. Denote the mean and variance of $W_{s}$ by $m_{n}$ and $s_{n}^{2}$, respectively. According to [19], the random variable $\left(W_{s}-m_{n}\right) / s_{n}$ will converge to the Gaussian distribution. The system performance weight $W_{s}$ will converge to the Gaussian distribution $N\left(m_{n}, s_{n}^{2}\right)$, where $N\left(m_{n}, s_{n}^{2}\right)$ is the Gaussian distribution with expect value $m_{n}$ and variance $s_{n}^{2}$

With the assumption of independence between system components, the mean value $m_{n}$ and the variance $s_{n}^{2}$ can be calculated directly from the mean value $\mu_{i}(i=1,2, \ldots, n)$ and the variance $\sigma_{i}^{2}(i=1,2, \ldots, n)$ of component $i$ :

$$
m_{n}=\sum_{i=1}^{n} \mu_{i} \quad \mathrm{~s}_{n}^{2}=\sum_{i=1}^{n} \sigma_{i}^{2}
$$

And the mean value $\mu_{i}$ and the variance $\sigma_{i}^{2}$ of component $i$ can be evaluated by Eq. (2).

$$
u_{i}=\sum_{i=1}^{s_{i}} p_{i, j} \cdot w_{i, j} \quad \sigma_{i}^{2}=\sum_{i=1}^{s_{i}} p_{i, j} \cdot\left(w_{i, j}-u_{i}\right)^{2}
$$

where $s_{i}$ is the number of states of component $i . w_{i, j}$ is the performance weight of component $i$ with the corresponding probability $p_{i, j}$ in state $j\left(j=1,2, \ldots, s_{i}\right)$.

Therefore, the approximate reliability $\tilde{R}\left(k^{*}\right)$ of a MSWKNS satisfying the given system demand $k^{*}$ can be approximated as [19]:

$$
\begin{aligned}
\tilde{R}\left(k^{*}\right) & =\operatorname{Pr}\left(W_{s} \geq k^{*}\right)=\operatorname{Pr}\left(\left(W_{s}-m_{n}\right) / s_{n} \geq\left(k^{*}-m_{n}\right) / s_{n}\right) \\
& =1-\operatorname{Pr}\left(\left(W_{s}-m_{n}\right) / s_{n}<\left(k^{*}-m_{n}\right) / s_{n}\right) \\
& \approx 1-\Phi\left(\left(k^{*}-m_{n}\right) / s_{n}\right)
\end{aligned}
$$


where $\Phi$ is the cumulative probability function of a standard normal distribution, whose calculation process is summarized in [19].

In structure screening, there are $N$ MSWKNS structure designs $S_{i}(i=1,2, \ldots, N)$. By employing CDA to all the MSWKNS structures, approximate reliabilities $\tilde{R}\left(k^{*}\right)$ of MSWKNSs can be evaluated. These MSWKNS structures with highest approximate reliabilities are picked out into next steps by OO algorithm.

\subsection{Ordered performance curve for N MSWKNSS}

As we know, "order" is easier than "value" [15]. It is much easier to estimate whether design $\mathrm{A}$ is better than $\mathrm{B}$ than to estimate the difference between A and B. Moreover, nothing but the best is very costly [15]. The idea of relaxing the goal of performance evaluation from "nothing but the best" to "settle for anything in the top choices" can buy quite a bit in the easing of computational burden. It is noticeable that to quickly narrow the search for optimal structure to a "good enough" subset is more important than to estimate accurately the performances of systems in structure screening. OO can solve the computational problem by comparing "order" first and estimating "value" second [15]. Therefore, OO algorithm is adopted to improve the computation efficiency.

Based on CDA, the approximate reliabilities $\tilde{R}\left(k^{*}\right)=\left\{\tilde{R}_{1}\left(k^{*}\right), \tilde{R}_{2}\left(k^{*}\right), \ldots, \tilde{R}_{N}\left(k^{*}\right)\right\}$ of all the MSWKNS structure designs $S=\left\{S_{1}, S_{2}, \ldots, S_{N}\right\}$ have been evaluated. These approximate reliabilities are plotted from the smallest to the largest to form a nondecreasing curve, which is named as ordered performance curve (OPC) in OO. There are five basic classes for OPCs, Steep, U-Shaped, Neutral, Bell and Flat, respectively [16]. The OPC in structure screening can be seen in Fig. 3. In Fig. 3, the $x$-axis represents the order of $N$ MSWKNS structures. The $y$-axis shows its corresponding approximate reliabilities. The five solid curves represent the five basic classes of OPCs, which are Steep, Bell, Neutral, U-Shaped and Flat, respectively. 
The class of OPC determines the value of screening coefficients $z_{0}, \rho, \eta$ and $\gamma$ [15]. The relationship between coefficients and the classes of OPCs are shown in Appendix [15]. Together with screening rule mentioned below, these coefficients decide the number of top MSWKNS structure designs with highest approximate reliabilities that can be selected into next steps.

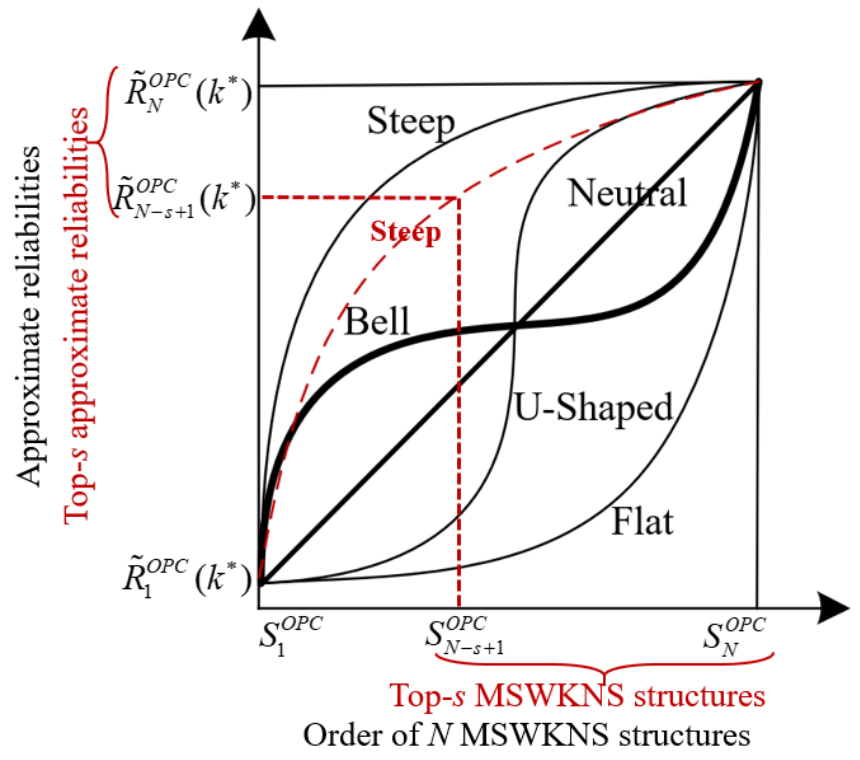

Fig. 3 OPC in structure screening.

After sorting the approximate reliabilities of $N$ MSWKNS structures from smallest to largest, the new order of $N$ MSWKNS structure designs $S^{O P C}$ and its corresponding approximate reliabilities $\tilde{R}^{O P C}\left(k^{*}\right)$ are noted as:

$$
S^{O P C}=\left\{S_{1}^{O P C}, S_{2}^{O P C}, \ldots, S_{N}^{O P C}\right\}
$$

(4)

$$
\tilde{R}^{O P C}\left(k^{*}\right)=\left\{\tilde{R}_{1}^{O P C}\left(k^{*}\right), \tilde{R}_{2}^{O P C}\left(k^{*}\right), \ldots, \tilde{R}_{N}^{O P C}\left(k^{*}\right)\right\}
$$

And the reliabilities satisfy the condition:

$$
\tilde{R}_{1}^{O P C}\left(k^{*}\right) \leq \tilde{R}_{2}^{O P C}\left(k^{*}\right) \leq \ldots \leq \tilde{R}_{N}^{O P C}\left(k^{*}\right)
$$


where $S_{i}^{O P C}$ is the MSWKNS which has the $(N-i+1)^{\text {th }}$ largest approximate reliability $\tilde{R}_{i}^{O P C}\left(k^{*}\right)$ among all the MSWKNSs.

Based on the orders above, the OPC of MSWKNS structures can be plotted. According to the shape of OPC made by approximate reliabilities in structure screening, the related class of OPC can be decided by comparing it to the five basic OPCs. For instance, if the OPC by approximate reliabilities is like the dotted red curve in Fig. 3, its related OPC class can be considered as Steep, as it illustrates the same tendency as the basic class of Steep. Once the class of OPC in structure screening is determined, its corresponding screening coefficients can be decided as well. These coefficients are utilized into next steps with the structure screening rule.

\subsection{Structure screening rule}

In $\mathrm{OO}$ algorithm, selected subset is defined, which is the set of selected designs in $N$ MSWKNS structures (usually the estimated top- $s$ of $N$ ). Every optimization problem can be conceived as the goal of matching a selected subset with the searching space. The degree of matching is called the alignment level $k$, and the confidence of achieving a certain alignment level is referred as the alignment probability $\alpha$ [16]. $k$ is a predefined value by engineering and $\alpha$ is no less than 0.95 in OO [16]. It is noted that the value of $s$ indicates how large the selected subset must be to guarantee that selected subset contains at least $k$ superior designs with high probability. Therefore, the selection of top- $s$ MSWKNS structures is very significant. The structure screening rule is used to determine the size of selected subset using the screening coefficients calculated by the estimated crude performance [16].

In this paper, we apply Horse Racing rule (HR) as the structure screening rule. The HR can be pictured as having all designs competing at the same time, similar to $g$ horses running a race. The positions of the designs are determined by their estimated performance values [15]. 
HR is used to determine the value of $s$, which can be formulated as Eq. (6). Eq. (6) gives suggestions on the subset size $s$ when the alignment level $k$ and class of OPC are given.

$$
s=\left\lceil e^{z_{0}} \cdot k^{\rho} \cdot g^{\gamma}+\eta\right\rceil
$$

where $z_{0}, \rho, \eta$ and $\gamma$ are constants depending on the OPC. $g$ is the number of MSWKNS structure designs.

In structure screening, the approximate reliabilities $\tilde{R}^{O P C}\left(k^{*}\right)$ of $N$ MSWKNSs $S^{O P C}$ are plotted from the smallest to the largest. The corresponding screening coefficients are determined by the related class of OPC, such as the dotted red curve in Fig. 3. The value of $s$ in structure screening can be calculated by Eq. (6). The top-s MSWKNS structures with higher approximate reliabilities are selected from the $N$ MSWKNSs, which can be seen in Fig.

3. The new ascending order of approximate reliabilities $\tilde{R}^{T O P}\left(k^{*}\right)$ and its corresponding top-s MSWKNS structures $S^{T O P}$ are characterized as:

$$
S^{T O P}=\left\{S_{1}^{T O P}, S_{2}^{T O P}, \ldots, S_{s}^{T O P}\right\}
$$

$$
\tilde{R}^{T O P}\left(k^{*}\right)=\left\{\tilde{R}_{1}^{T O P}\left(k^{*}\right), \tilde{R}_{2}^{T O P}\left(k^{*}\right), \ldots, \tilde{R}_{s}^{T O P}\left(k^{*}\right)\right\}
$$

where $S_{i}^{T O P}$ is the MSWKNS which has approximate reliability $\tilde{R}_{i}^{T O P}\left(k^{*}\right)$ among the top-S $(s \leq N)$ MSWKNSs.

By applying HR in structure screening, the top- $s$ systems are chosen in selected subset. The optimal MSWKNS structure is selected from these top- $s$ systems considering system cost and exact reliability constraints. We only need to evaluate the exact reliabilities and system cost of $s$ MSWKNSs, which is less than the number of $N$. The selected MSWKNS structures $S^{T O P}$ are employed into next steps with UGF technique. 


\subsection{Exact reliabilities of $s$ MSWKNSs}

After the application of CDA and OO algorithm, the top-s MSWKNS structures $S^{T O P}$ are selected among $N$ structure designs. As the reliability of optimal system structure should satisfied the reliability constricts, the exact reliabilities of these selected systems should be evaluated. UGF technique is utilized to exact reliability assessment. In OSS method, the computational burden for reliability evaluation is dramatically reduced as only $s$ MSWKNSs that need to evaluate.

Based on the definitions of components mentioned above, the UGF of a component states associating different weights $w_{i, j}$ with its probability $p_{i, j}$ is $u_{i}(z)=\sum_{j=1}^{k_{i}} p_{i, j} \cdot z^{w_{i, j}}$. Applied on the UGF of $n$ components, the UGF of a MSWKNS is calculated by the $\Omega_{\phi}$ composition operator and $\phi_{P}$ structure function [10].

$$
\begin{aligned}
U(z) & =\Omega_{\phi}\left(u_{1}(z), \cdots, u_{n}(z)\right) \\
& =\sum_{j_{1}=1}^{k_{1}} \cdots \sum_{j_{n}=1}^{k_{n}}\left(\prod_{i=1}^{n} p_{i, j_{i}} z^{\phi_{P}\left(w_{1, j_{1}}, \cdots, w_{n, j_{n}}\right)}\right)
\end{aligned}
$$

The structure function $\phi_{P}$ is defined as [10]:

$$
\phi_{P}\left(W_{1}, \cdots, W_{n}\right)=\sum_{i=1}^{n} W_{i}
$$

Based on the UGF of the whole MSWKNS, the exact reliability $R\left(k^{*}\right)$ is evaluated by Eq. (11).

$$
R\left(w^{*}\right)=\operatorname{Pr}\left(W_{s} \geq k^{*}\right)=\sum_{i=1}^{K} p_{i} \cdot \chi\left(w_{i}-w^{*}\right)
$$

where $K$ is the number of states of a system with its UGF representation $U(z)=\sum_{i=1}^{K} p_{i} z^{w_{i}}$. $p_{i}$ is the probability associated with the MSWKNS in state $i$ with performance weight $w_{i}$. The function $\chi$ is defined as the step function $\chi(x)=1(x \geq 0)$, which equals to 0 if the variable $x$ is less than 0 and equals to 1 otherwise [10] 
By employing UGF technique to the selected top- $s$ MSWKNSs, the set of exact reliabilities of $S^{T O P}$ is evaluated, which is represented by $R\left(k^{*}\right)=\left\{R_{1}\left(k^{*}\right), R_{2}\left(k^{*}\right), \ldots, R_{s}\left(k^{*}\right)\right\}$. The feasible MSWKNS structure designs whose exact reliabilities are no less than the required reliability $R^{*}$ can be screened. Assume that the number of feasible MSWKNS structure designs is $q(q \leq s)$. Thus, the set of all feasible MSWKNS structure designs $S^{U G F}$ and its corresponding exact reliabilities $R\left(k^{*}\right)$ are formed as:

$$
S^{U G F}=\left\{S_{1}^{U G F}, S_{2}^{U G F}, \ldots, S_{g}^{U G F}\right\}
$$

$$
R\left(k^{*}\right)=\left\{R_{1}\left(k^{*}\right), R_{2}\left(k^{*}\right), \ldots, R_{g}\left(k^{*}\right)\right\}
$$

where $S_{i}^{U G F}$ is the MSWKNS structure whose exact reliability is $R_{i}\left(k^{*}\right)$.

\subsection{Optimal system among $q$ MSWKNSs}

The $q$ feasible MSWKNS structure designs $S^{U G F}$ are selected by UGF. Each MSWKNS structure $S_{i}^{U G F}$ has $M_{i}$ versions of components $V_{i, j}\left(j=1,2, \ldots, M_{i}\right)$. The total number of components in version $j V_{i, j}$ is $n_{i, j}$ and the purchase cost for a component in $V_{i, j}$ is $c_{i, j}$. $C_{i}^{C O S T}$ is the system cost for MSWKNS $S_{i}^{U G F}$, which can be evaluated by Eq. (14). The set of the system costs for all feasible MSWKNS structure designs $S^{U G F}$ is characterized as $C^{\operatorname{COST}}=\left\{C_{1}^{\operatorname{COST}}, C_{2}^{\operatorname{COST}}, \ldots, C_{g}^{\operatorname{COST}}\right\}$

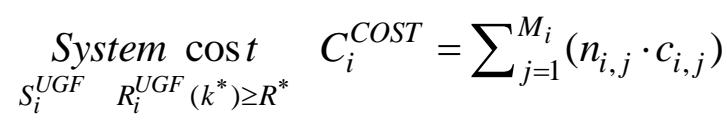

According to the calculated system costs $C^{\operatorname{COST}}$, the optimal MSWKNS structure with minimal system cost under reliability constraints can be determined.

\subsection{OSS method}


Based on OO, UGF, CDA and structure screening rule mentioned above, OSS method in structure screening is formulated. The procedure of the OSS method is illustrated in Fig. 4.

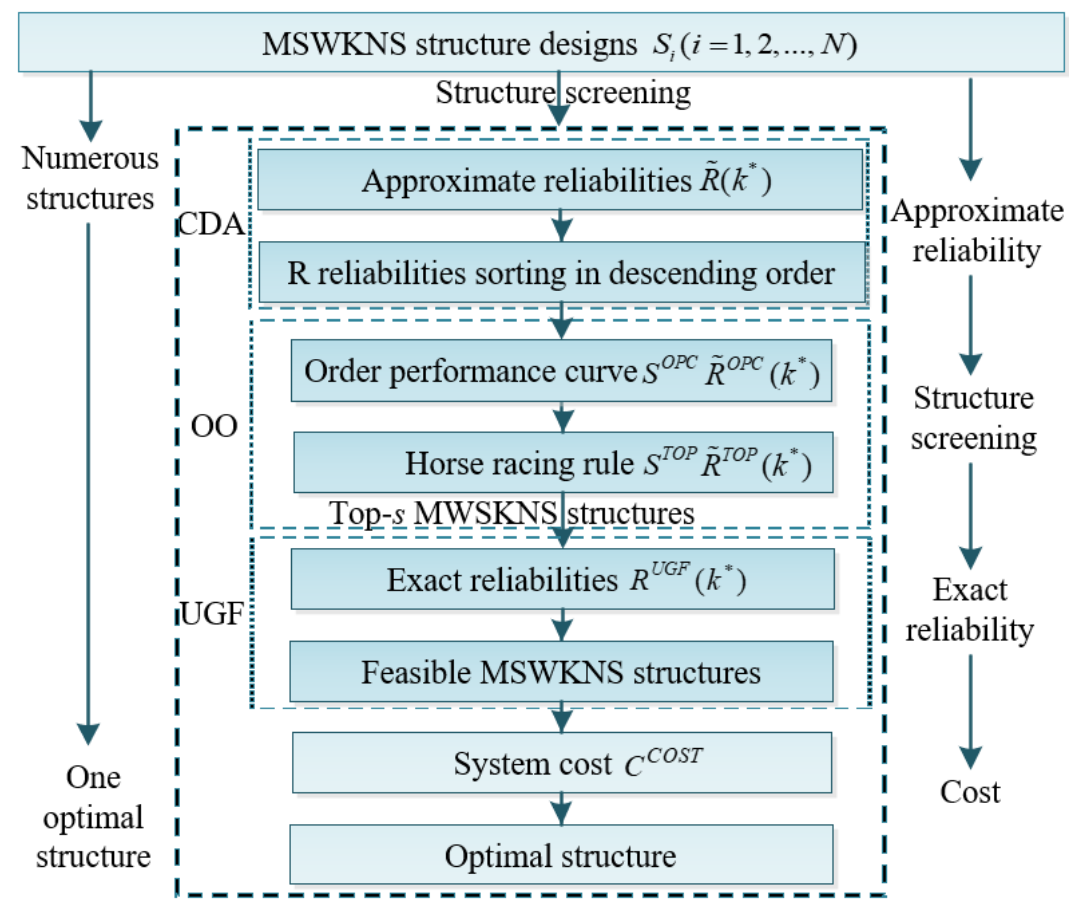

Fig. 4 Flow chart of OSS.

Step 1) Approximate reliabilities evaluation: Apply CDA to evaluate the approximate reliabilities $\tilde{R}\left(k^{*}\right)$ of $N$ MSWKNS structure designs by Eq. (3).

Step 2) Ordered performance curve: Determine the class of OPC and the values of $z_{0}, \rho$, $\eta$ and $\gamma$ by sorting the approximate reliabilities $\tilde{R}^{O P C}\left(k^{*}\right)$ in ascending order.

Step 3) Structure screening rule: Apply HR rule in structure screening to determine the top-s MSWKNS structures $S^{T O P}(s \leq N)$ with highest approximate reliabilities $\tilde{R}^{O P C}\left(k^{*}\right)$.

Step 4) Exact reliabilities evaluation: Evaluate the exact reliabilities $R\left(k^{*}\right)$ of the selected top- $s$ MSWKNS structures by UGF technique and screen the $g$ feasible MSWKNS structure designs $S^{U G F}(g \leq s)$ whose exact reliabilities $R\left(k^{*}\right)$ are no less than the required reliability.

Step 5) Optimal system determination: Calculate the system cost $C^{\operatorname{Cos} T}$ by Eq. (14) and determine the optimal system among the feasible MSWKNS structure designs $S^{U G F}$. 


\section{Numerical Studies}

\subsection{Case 1: The standard MSWKNS}

This example is to illustrate the proposed method in detail and analyze the accuracy and efficiency of OSS.

We consider the system structure composed of 15 components. There are five versions of component, presented as $v_{i}(i=1,2, \ldots, 5)$. The performances of components in version $v_{i}$ is measured by its purchase component $\operatorname{cost} c_{i}$, probability $p_{i, j}$ and performance weight $w_{i, j}$ corresponding to state $j$, respectively. The parameters of components from five versions are list in Table 1. The component cost from different versions is equal to $0.8,0.9,0.95,0.74$ and 1, respectively [21]. Moreover, the total number of MSWKNS structures applied in this case is equal to 28 . The system required demand is 5. Suppose the required reliability is 0.88 . Structure of each MSWKNS can be seen in Fig. 5. In Fig. 5, the $x$-axis represents the order of component versions and the $y$-axis shows the number of components in each version.

Table 1 Parameters of components from five versions

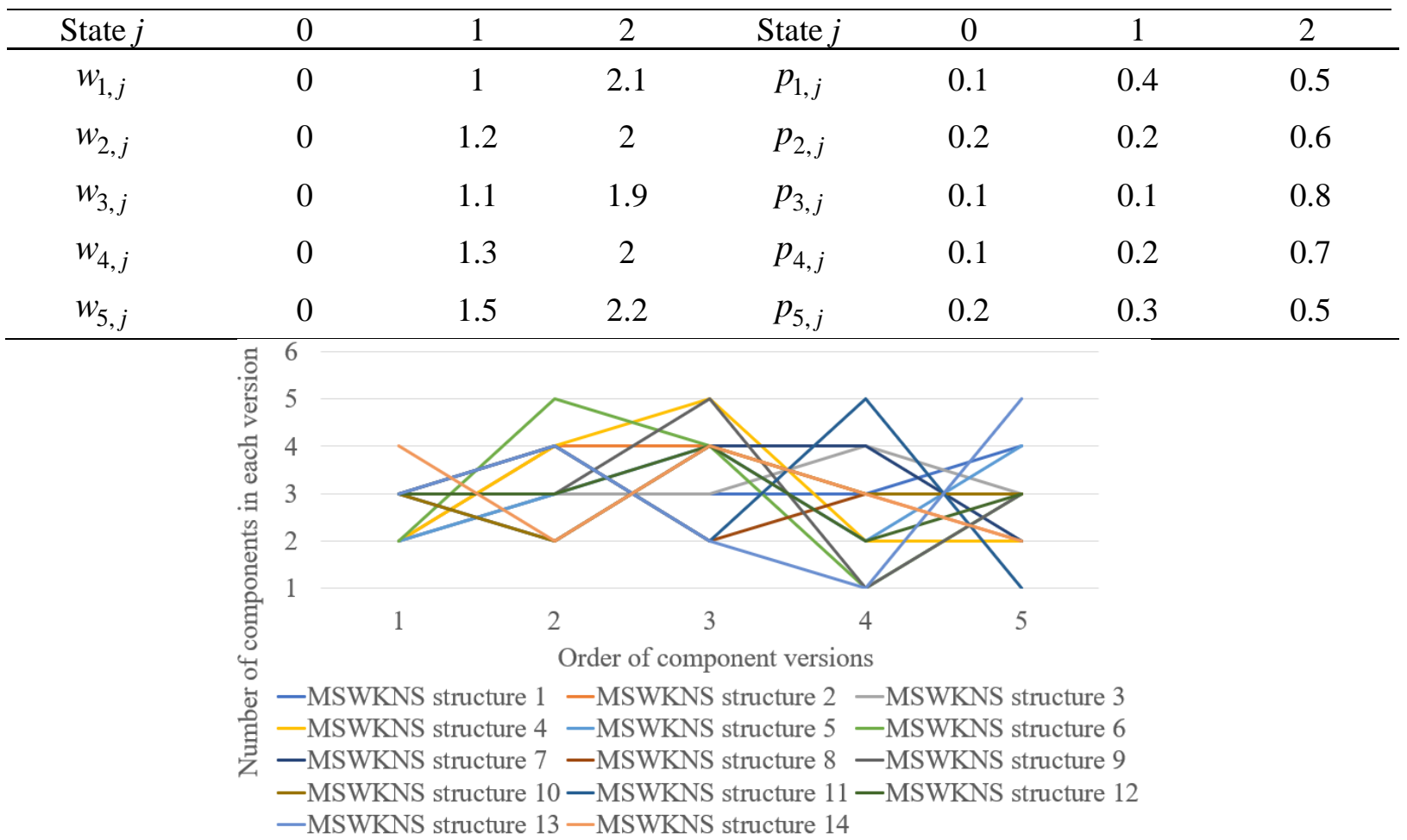


(a)

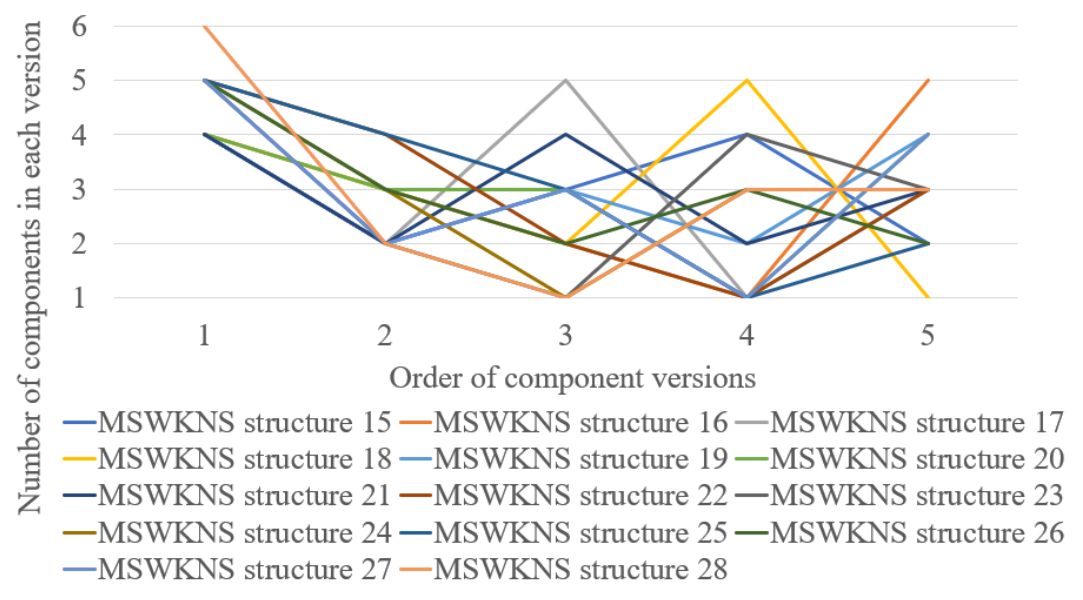

(b)

Fig. 5 MSWKNS structures in case 1. (a) Structure 1 to structure 14. (b) Structure 15 to structure 28.

\subsubsection{Results of OSS method}

Approximate reliabilities of $28 \mathrm{MSWKNS}$ structure designs are evaluated by CDA. The difference between the accurate reliabilities calculated by UGF and the approximate reliabilities can be used as a measure of the error performance, which can be seen in Fig. 6. In Fig. 6, the $x$-axis represents the reliability order of structure designs. The system structure with higher order is the system with higher reliability. The $y$-axis shows the reliabilities by two methods. The blue and green bar charts are the exact reliabilities by UGF and the approximate reliabilities by CDA, respectively. It can be noticed from Fig. 6 that two bar charts are almost coincident, which means that the reliabilities evaluated by CDA method is in good approximation having high accuracy performance. 


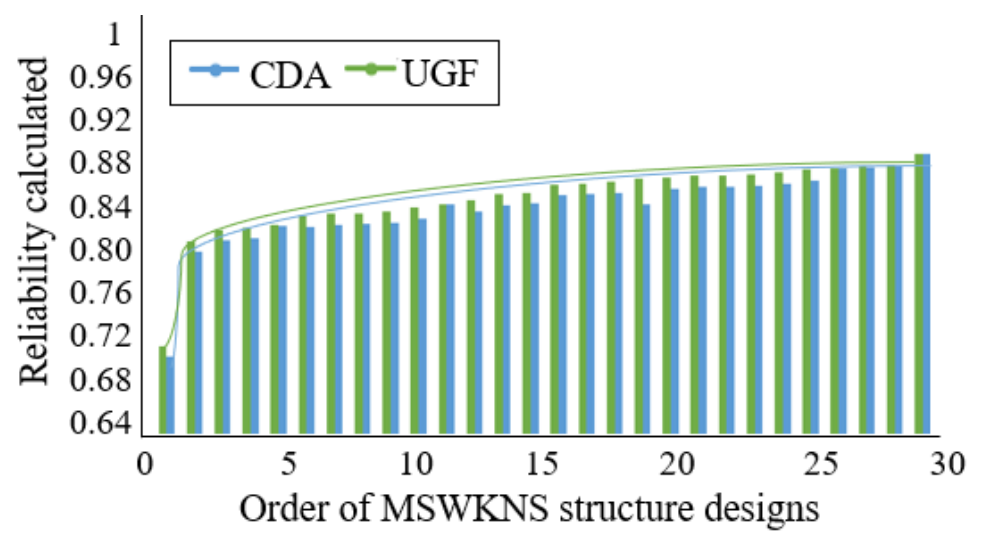

Fig. 6 System reliabilities obtained by different techniques

The class of OPC in this case is Steep based on the trend line of CDA in Fig. 6. Thus, the value of $z_{0}, \rho, \gamma$ and $\eta$ are 7.7998, 1.5099, -2.0719, 10, respectively [17]. $\alpha \%$ and $k$ are 95\% and 1 in this case, respectively. Then $s$ is calculated as 15 by Eq. (6), which means top15 MSWKNS structures should be sorted into selected subset. Finally, cost and exact reliabilities for top-15 MSWKNS structures are evaluated, which can be seen in Table 2. MSWKNS structure 18 can be selected as optimal structure which has minimal system cost and desirable reliability.

Table 2 Exact reliabilities and cost for MSWKNSs in selected subset

\begin{tabular}{cccccc}
\hline System & Reliability & Cost & System & Reliability & Cost \\
\hline 1 & 0.8779 & 13.37 & 12 & 0.8722 & 13.38 \\
2 & 0.8815 & 13.22 & 14 & 0.8861 & 13.02 \\
3 & 0.8886 & 13.11 & 15 & 0.8873 & 12.81 \\
4 & 0.8804 & 13.43 & 17 & 0.8731 & 13.49 \\
5 & 0.8786 & 13.58 & 18 & 0.8830 & 12.50 \\
7 & 0.9004 & 12.96 & 19 & 0.8648 & 13.33 \\
10 & 0.8898 & 13.22 & 21 & 0.8745 & 13.28 \\
11 & 0.8805 & 12.60 & & & \\
\hline
\end{tabular}

\subsubsection{Accuracy and efficiency comparison between UGF and OSS methods}

Comparisons between OSS and UGF technique are demonstrated in this part in terms of accuracy and computational time.

Table 3 Exact reliabilities and cost for all the MSWKNSs

\begin{tabular}{cccccc}
\hline System & Reliability & Cost & System & Reliability & Cost \\
\hline 1 & 0.8779 & 13.37 & 15 & 0.8873 & 13.37 \\
2 & 0.8815 & 13.22 & 16 & 0.8355 & 13.22 \\
3 & 0.8886 & 13.11 & 17 & 0.8731 & 13.11 \\
\hline
\end{tabular}




\begin{tabular}{cccccc}
\hline 4 & 0.8804 & 13.43 & 18 & 0.8830 & 13.43 \\
5 & 0.8786 & 13.58 & 19 & 0.8648 & 13.58 \\
6 & 0.8509 & 13.64 & 20 & 0.8453 & 13.64 \\
7 & 0.9004 & 12.96 & 21 & 0.8745 & 12.96 \\
8 & 0.8575 & 13.12 & 22 & 0.8204 & 13.12 \\
9 & 0.7243 & 13.59 & 23 & 0.8636 & 13.59 \\
10 & 0.8898 & 13.22 & 24 & 0.8436 & 13.22 \\
11 & 0.8805 & 12.60 & 25 & 0.8309 & 12.60 \\
12 & 0.8722 & 13.38 & 26 & 0.8543 & 13.38 \\
13 & 0.8327 & 13.64 & 27 & 0.8473 & 13.64 \\
14 & 0.8861 & 13.02 & 28 & 0.8458 & 13.02 \\
\hline
\end{tabular}

We apply UGF technique to evaluate the exact reliabilities for all the MSWKNS structures and select the feasible structures whose exact reliabilities are no less than the required one. Then calculate the system cost for all the feasible MSWKNS structures. The results are presented in Table 3. It can be noticed from Table 3 that structure 18 can be considered as optimal MSWKNS, which is the same result as OSS. Thus, OSS method presents its computational accuracy in structure screening.

Moreover, experiment below shows the efficiency of OSS method in reducing the number of reliability evaluation systems. The component in this comparison is treated as a multi-state component, whose number of states is varying from 2 to 5 . The versions of components for each structure are five. Moreover, the total number of components is also enlarged to demonstrate the efficiency of OSS. The experiments were developed by MATLAB2017b, in a computer with $2.60 \mathrm{GHz} \mathrm{CPU}$ and $4 \mathrm{~GB}$ of RAM. The computational time for OSS and UGF methods is compared in Table 4 and Fig. 7. In Fig. 7, the $x$-axis represents the number of states a component has. The $y$-axis reflects the computational time by UGF and OSS, respectively.

Table 4 CPU time for two methods

\begin{tabular}{cccc}
\hline $\begin{array}{c}\text { Total number of components } \\
\text { a MSWKNS has }\end{array}$ & $\begin{array}{c}\text { Number of states a } \\
\text { component has }\end{array}$ & Method & CPU time (seconds) \\
\hline \multirow{2}{*}{20} & 2 & UGF & 59 \\
& 3 & OSS & 20 \\
& & UGF & 2154 \\
& 4 & OSS & 705 \\
& 5 & UGF & 9586 \\
& & UGF & 3199
\end{tabular}




\begin{tabular}{cccc} 
& & OSS & 7905 \\
\hline & \multirow{2}{*}{30} & UGF & 308 \\
& & OSS & 85 \\
& \multirow{2}{*}{3} & UGF & 9540 \\
& & OSS & 2997 \\
& 4 & UGF & --- \\
\hline
\end{tabular}

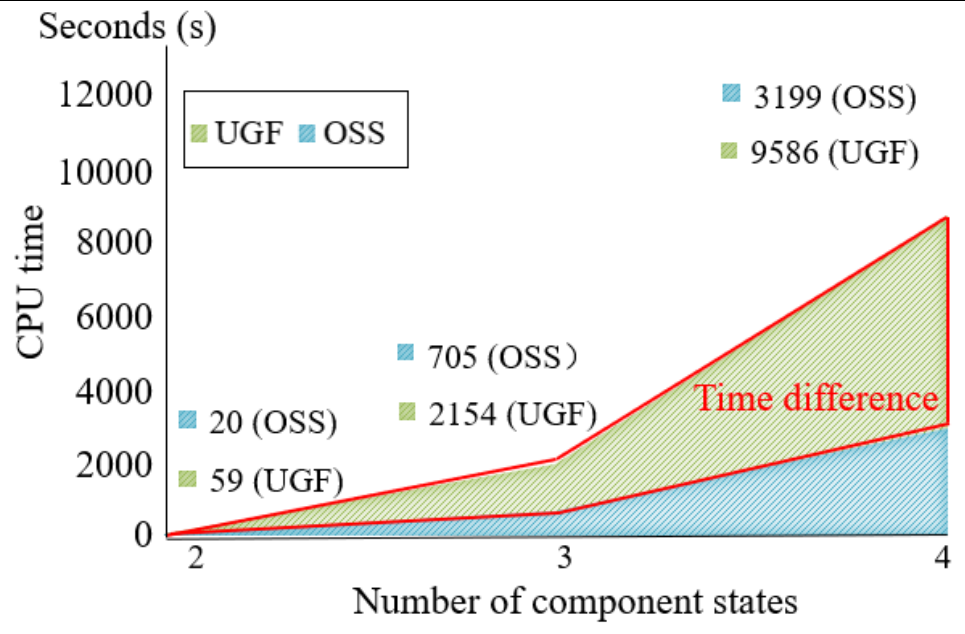

Fig. 7 Calculation time when the number of components is 20

From Table 4 and Fig. 7, it can be indicated that the calculation time of OSS is nearly onethird the time of UGF. Therefore, OSS method can effectively shorten the computational time, where its efficiency is more evident when the number of MSWKNS structures and system components are in large scale.

\subsection{Case 2: The practical power system}

This case is focused on the application of OSS in an electric power system, which is a typical example of MSWKNW. A typical electric power generation system consists of various multi-state generating units, and has different demand levels. Therefore, an electric power generation system can be represented as a MSWKNS.

Table 5 Parameters of components from five versions

\begin{tabular}{cccccccccc}
\hline State $j$ & 0 & 1 & 2 & 3 & State $j$ & 0 & 1 & 2 & 3 \\
\hline$w_{1, j}$ & 0 & 100 & 120 & 180 & $p_{1, j}$ & 0.1 & 0.3 & 0.3 & 0.3 \\
$w_{2, j}$ & 0 & 120 & 140 & 250 & $p_{2, j}$ & 0.2 & 0.4 & 0.2 & 0.2 \\
$w_{3, j}$ & 0 & 90 & 120 & 170 & $p_{3, j}$ & 0.1 & 0.1 & 0.4 & 0.4 \\
\hline
\end{tabular}


The total number of electric power generation system structure designs applied in this case is equal to 40. Suppose each generation system has 15 generating units from three versions: coal units, gas unit, and oil unit. The parameters of generating units from three versions are list in Table 5. The purchase cost for each version of generation unit is equal to $\$ 200$ million, $\$ 250$ million and \$320 million, respectively [21]. The number of components in coal units, gas unit, and oil unit of each structure design is varying from one to eight, two to seven, one to seven, respectively. The system load is 80 and the required reliability is 0.90 .

According to OSS method mentioned in Section 3 and the class of OPC made by approximate reliabilities of 40 MSWKNS structure designs, we choose top-18 MSWKNS structures with higher reliabilities in selected subset. The value of 18 is calculated by screening coefficients with $\alpha \%$ equal to $95 \%$ and $k$ equal to 2 . Finally, based on the exact reliabilities and system costs for those top-18 systems. MSWKNS structure 30 can be considered as the optimal structure, whose components in the three versions are 5, 4 and 6 , respectively.

The comparisons of accuracy and efficiency between OSS and UGF techniques in case 2 are provided in this part. The experiments were developed by MATLAB2017b, in a computer with $2.60 \mathrm{GHz}$ CPU and 4 GB of RAM. We apply UGF to evaluate the exact reliabilities and system costs for each MSWKNS structure designs. It is calculated that MSWKNS structure 30 can be treated as optimal structure, which is the same as OSS method. Moreover, the computational time for OSS is 48 minutes which is one-third the time of UGF technique with 2.5 hours. Therefore, in case 2, OSS method reveals its highly screening accuracy and efficiency for the screening among numerous MSWKNS structure designs.

\section{Conclusions}

In this work, structure screening of large-scale MSWKNS structure designs considering reliability constraints has been discussed. However, evaluating the exact reliabilities of large- 
scale MSWKNS structures requires expensive calculation cost and unlimited computational resource, particularly when the number of MSWKNS designs increases. To efficiently and accurately determine the optimal one among numerous structure designs, OSS method has been utilized in this paper. Adopted the central limit theory, CDA is used to approximate the reliability of a large-scale MSWKNS, which can accelerate the reliability evaluation process. The structure screening rule in $\mathrm{OO}$ algorithm is applied as a desirable tool to limit the searching space, which can effectively reduce the number of large-scale structure designs. And finally, UGF is employed to evaluate the exact reliabilities when the number of structures has been greatly shorted by the above steps. The results show that there is no significant difference between the accuracy of OSS and traditional structure screening methods. Moreover, OSS method can reduce the computational resources among large-scale MSWKNS structures.

\section{Appendix}

Table 6 Values of screening coefficients

\begin{tabular}{cccccc}
\hline OPC class & Flat & U-Shape & Neutral & Bell & Steep \\
\hline$z_{0}$ & 8.1378 & 8.1200 & 7.9000 & 8.1998 & 7.7998 \\
$\rho$ & 0.8974 & 1.0044 & 1.0144 & 1.9164 & 1.5099 \\
$\gamma$ & -1.2058 & -1.3695 & -1.3995 & -2.0250 & -2.0719 \\
$\eta$ & 6.00 & 9.00 & 7.00 & 10.00 & 10.00 \\
\hline
\end{tabular}

Acknowledgements

This work was supported partly by the National Natural Science Foundation of China (Grant \#71871200 and \#51537010).

\section{References}

[1]. A. Lisnianski, G. Levitin, "Multi-state system reliability: assessment, optimization and applications," New York, NY, USA: World Scientific, 2003.

[2]. Y. Mo, L. Xing, S V, Amari, "Efficient analysis of multi-state k -out-of- n, systems," Rel. Eng. Syst. Safety, vol.133, no.133, pp.95-105, 2015

[3]. Y. Ding, M. Zuo, Z. Tian, "The hierarchical weighted multi-state, $k$-out-of- $n$ System model and its application for infrastructure management," IEEE Trans.Rel., vol.59, no.3, pp. 593-603, 2010.

[4]. S.C. Horng, S.S. Lin, F.Y. Yang, "Evolutionary algorithm for stochastic job shop scheduling with random processing time," Expert. Syst. Appl., vol.39, no.3, pp. 3603-3610, 2012. 
[5]. S.C. Horng, F.Y. Yang, S.S. Lin, "Apply PSO and OCBA to minimize the overkills and re-probes in wafer probe testing," IEEE Trans. Semicond. Manuf., vol.25, no.3, pp. 531-540, 2012.

[6]. J. Mi, Y. Li, Y. Liu, "Belief universal generating function analysis of multi-state systems under epistemic uncertainty and common cause failures," IEEE Trans.Rel., vol. 64, no.4, pp. 1300-1309, 2015.

[7]. S. Si, G. Levitin, H. Dui, "Component state-based integrated importance measure for multi-state systems," Rel. Eng. Syst. Safety,vol.116, pp. 75-83, 2013.

[8]. W. Li and M. J. Zuo, "Optimal design of multi-state weighted k-out-of-n systems based on component design,” Rel. Eng. Syst. Safety, vol. 93, no. 11, pp. 1673-1681, 2008.

[9]. I. Ushakov, “Universal generating function”, Soviet J. Computer System Science, vol. 24, no. 5, pp. 118-129, 1986.

[10].G. Levitin, "Universal generating function and its applications,” New York: Springer, 2005.

[11].E. Zio, "The Monte carlo simulation method for system reliability and risk analysis," New York: Springer, 2013.

[12].W. Li, "Reliability assessment of electric power systems using Monte Carlo methods," London: Springer, 2013.

[13].M. Perman, A. Senegacnik and M. Tuma, "Semi-markov models with an application to power-plant reliability analysis," IEEE Trans. Rel., vol. 46, no. 4, pp. 526-532, 1997.

[14].Y. Ho, L. Lee, E. Lan, "Explanation of goal softenign in ordinal optimization," IEEE Trans. Automat. Contr., vol. 44, no.1, pp. 94-99, 1999.

[15].Y. Ho, Q. Zhao, Q. Jia, “Ordinal optimization: soft optimization for hard problems,” Berlin, Germany: Springer,2006.

[16].J. Zhang, C. Wang, D. Zang, "Incorporation of ordinal optimization into learning automata for high learning efficiency,"in Proc. IEEE. Int. Conf. Automa.Sci.Eng., pp. 1206-1211, 2015.

[17].S. Hong, S. Lin, "Ordinal optimization based metaheuristic algorithm for optimal inventory policy of assemble-to-order systems," Appl. Math. Model., vol.42, pp. 43-57, 2017.

[18].S. Nanchian, A. Majumdar, B. Pal, "Ordinal optimization technique for three phase distribution network state estimation including discrete variables," IEEE Trans.Sustain.Energy., vol.8, no.4, pp. 1528-1535, 2017.

[19].Y. Ding, Y. Lin, R. Peng, M. Zuo, “Approximate reliability evaluation of large-scale multi-state seriesparallel system,” IEEE Trans.Rel., online, 2019, DOI: 10.1109/TR.2019.2898459.

[20].M.G. Charles and J. Laurie Snell, "Introduction to Probability," 2nd ed., American Mathematical Society, 2003.

[21].Y. Ding, M. Zuo, A. Lisnianski, “A framework for reliability approximation of multi-state weighted k-outof-n Systems,” IEEE Trans.Rel., vol. 59, no. 2, pp. 297-308, 2010 